Aus dem Zentralen Röntgeninstitut des Rätischen Kantons- und Regionalspitals Chur

\title{
Alexander Rzewuski und die Anfänge der Radiologie in der Schweiz
}

Zum 75jährigen Jahrestag der Entdeckung der Röntgenstrahlen

Von C. Wieser und U. Keller

Wenige Entdeckungen der theoretischen Physik haben so rasch und tiefgreifend die moderne Medizin umgestaltet wie jene der X-Strahlen durch Wilhelm Connad Röntgen.

Am Abend des 8. November 1895 beobachtete Rönten, damals Professor der Physik an der Universität Würzburg, beim Experimentieren mit Kathodenstrahlen eine ungewöhnliche Naturerscheinung. Obwohl die Röhre, mit welcher er die Kathodenstrahlen erzeugte, mit schwarzem Karton abgeschirmt war, leuchteten in einiger Entfernung auf dem Tisch liegende Kristalle auf. In ebenso genialer wie gründlicher Weise ging Röntgen dieser Erscheinung nach und fand, daß sie durch bisher unbekannte Strahlen bewirkt wurde. Sieben Wochen später, am 28. Dezember 1895, überreichte er dem Sekretär der Physikalisch-Medizinischen Gesellschaft in Würzburg ein Manuskript «Über eine neue Art von Strahlen», das Ergebnis seiner Forschungen. Obwohl wegen der Festtage keine Sitzung der Gesellschaft stattfand, wurde diese "vorläufige Mitteilung» sofort in den Sitzungsberichten abgedruckt und erschien in den ersten Januartagen 1896 (21).

Noch bevor Röntgen selbst in seinem berühmten Vortrag vom 23. Januar 1896 vor der Physikalisch-Medizinischen Gesellschaft in Würzburg die Öffentlichkeit über die X-Strahlen informieren konnte, hatte die Wiener Tageszeitung Die Presse bereits am 5. Januar 1896 unter dem Titel «Eine sensationelle Entdeckung» darüber berichtet. RöNTGEN selber hatte in einem Brief an seinen Wiener Kollegen und Freund Professor Exner davon Mitteilung gemacht, der seinerseits einem kleinen Kreis von Fachkollegen und Bekannten die Beobachtungen des Würzburger Physikprofessors bekanntgab (26).

Mit erstaunlichem Weitblick wird in diesem Zeitungsbericht die volle Tragweite der Entdeckung erkannt und auf die Möglichkeit der Anwendung in der medizinischen Diagnostik bei Fremdkörperlokalisation, 
Frakturbehandlung, Erkennung von Knochen- und möglicherweise auch anderen Organkrankheiten hingewiesen.

Der erwähnte Bericht wurde von allen großen europäischen Tageszeitungen übernommen. So druckte bereits am 10. Januar 1896 die Neue Zürcher Zeitung den Wiener Originalartikel ab, versehen mit einer Einleitung, in der besonders auf den Triumph der «exakten kombinierenden Beobachtung» (kombiniert mit einem "glücklichen Zufall»), die "gelehrte Theorie» und das "philosophische Denken» hingewiesen wird (24).

Im Anschluß an diese Bekanntmachung in der Tagespresse hat sich die Öffentlichkeit brennend für die neu entdeckten Strahlen interessiert. Überall in der Welt wurden die Experimente RöNTgens wiederholt und die Anwendung der X-Strahlen praktisch erprobt. So wurde am Tage des eingangs erwähnten Würzburger Vortrages Röntgens in Wien bereits die erste Angiographie an einer Leichenhand publiziert (22)! Dagegen berichtet das Correspondenzblatt für Schweizer Ärzte erst am 15. Februar 1896 kurz über diese Entdeckung und die ersten Erfahrungen in der Schweiz (1).

\section{Erste Versuche in der Schweiz}

In Bern entfernte Professor Kocher Mitte Januar 1896 mit Hilfe einer Röntgenaufnahme, die ihm vom Physiker Professor Forster angefertigt worden war, eine Nadel aus einer Hand. Die Nachricht darüber erschien am 1.Februar 1896 in Lancet in London und entstammt einer Berliner Korrespondenz (persönliche Mitteilung von Herrn Professor E. Hintzsche). Am 27.Januar 1896 berichtete Forster vor dem medizinisch-pharmazeutischen Bezirksverein Bern anhand eigener Beobachtungen über die Röntgensche Entdeckung (5). In einer ersten Originalarbeit im Correspondenzblatt referieren C.F.Traczewski, O.Lenz und G.Lenz über ihre Versuche im Rahmen der Technischen Abteilung der Eidgenössischen Telegraphendirektion im Februar 1896. In erster Linie handelte es sich hier um Schußversuche an Leichen, durchgeführt in der Eidgenössischen Waffenfabrik, zum Studium der dabei entstandenen Verletzungen im Radiogramm, dann aber auch um Gefäßdarstellungen an Leichenpräparaten. Wesentlich ist die Diskussion von Artefakten (Filmfehlern!) und Überlagerungseffekten (Beschreibung des sogenannten Machschen Effektes bei Aufnahmen durch Gipsverbände).

In Zürich demonstrierte am 30. Januar 1896 im Rahmen eines klinischen Ärztetages Professor Pernet im Hörsaal des Physikalischen Institutes der Е тн die neuen Strahlen. 
«Rauschender Beifall lohnte die große Mühe und prachtvolle Demonstration. Zum Dank ernannte die Kantonale ärztliche Gesellschaft Prof. Dr.Pernet zum Ehrenmitglied und sandte ein Glückwunschtelegramm an Professor Röntgen in Würzburg.» (2)

Im Sommer 1896 existierte in Zürich in der Glühlampenfabrik Hard bereits ein «Röntgeninstitut», das «ganz gute Aufnahmen für Ärzte» beim ambulanten Patienten anfertigte (9).

Über einen ersten Vortrag vor der Medizinischen Gesellschaft der Stadt Basel berichtet das Correspondenzblatt in einer Mitteilung über die Sitzung vom 12. März 1896: Dr.A.Hägler-à Wengen referierte bei dieser Gelegenheit über eine Fremdkörperlokalisation mit Hilfe der Röntgenstrahlen (4).

Ebenfalls in Basel hielt Prof. Dr. Hagenbach-Bischoff anläßlich der Sitzung des ärztlichen Zentralvereins am 30. Mai 1896 ein großes Übersichtsreferat «Über die Röntgensche Entdeckung» (7).

Eine erste öffentliche Diskussion unter «Röntgenologen» der deutschen Schweiz fand anläßlich der Versammlung des ärztlichen Zentralvereins in Olten am 31.Oktober 1896 statt (9). Neben Mitteilungen über Fremdkörperlokalisation, Diagnostik von Frakturen und Luxationen, Beobachtungen von verkalkten Lungenherden sowie Erkennung von Mißbildungen des Skelettes wurden vor allem auch spezifisch röntgendiagnostische Probleme besprochen. Die Forderung nach zwei Aufnahmen in aufeinander senkrecht stehenden Ebenen wurde von einem «Röntgenpraktiker», dem Aargauer Bezirkslehrer Wust, erhoben, während Professor Forster zur Berechnung (!) der Lage des Fremdkörpers eine Doppelbelichtung mit Verschiebung der Lichtquelle vorschlug. Besonderen Wert wurde der röntgenologischen Verlaufsbeobachtung und der juristischen Bedeutung von Schlußkontrollen beigemessen. Spitalarzt Dr.H.Bircher, Aarau, «betont die Notwendigkeit, daß die Einrichtung und Besorgung des ganzen Verfahrens durch Fachleute besorgt werde».

\section{Alexander Rzewuski (1861-1949)}

In Davos besaß Alexander Rzewuski ein kleines Privatlaboratorium. Nach Absolvierung des Gymnasiums in Kattowitz und Gleiwitz war er 1879 wegen eines schweren Asthmaleidens nach Davos gekommen. Hier fand er Heilung und zugleich eine zweite Heimat. Guter Alpinist, hatte sich Rzewuski als Amateurphotograph einen Namen gemacht. Seine Platten entwickelte er selber und er hatte dazu ein eigenes Laboratorium eingerichtet. Hier führte der Privatgelehrte auch physikalische und chemische 
Experimente durch $(20,23)$. Durch die Zeitung muß auch Rzewuski auf Röntgens Entdeckung aufmerksam geworden sein. Denn in seiner Mitte August 1896 in der Naturwissenschaftlichen Rundschau erschienenen Arbeit über die «Chemische Wirkung der X-Strahlen» schreibt er: «Mit einem solchen (Crookesschen) Rohr habe ich anfangs Januar meine ersten Aufnahmen in 1 Stunde gemacht.» Es dürfte dies eines der ersten, wenn nicht sogar das erste in der Schweiz hergestellte Röntgenbild gewesen sein (23).

Zudem wissen die Davoser Blätter bereits am 15. Februar 1896, über einen durch zahlreiche Experimente veranschaulichten öffentlichen Vortrag über die epochenmachende Entdeckung Professor Röntgens im Saal des Davoser Conversationshauses durch Alexander Rzewuski zu berichten.

Dabei ging der Referent auch auf die bisherige Entwicklung der theoretischen Physik ein, welche Röntgens Entdeckung erst ermöglichte. «Denn unmittelbar hinter Röntgen stehen Lenard, Herz, Hittorf, Crookes und alle anderen Kathodenstrahlenforscher und mit ihnen wiederum alle Wissenschaftler und Techniker, die zur Erkenntnis der Eigenschaften der Elektrizität, der Erzeugung hochgespannter Ströme und des Durchganges dieser Ströme durch hochevakuierte Röhren beitrugen.»

Von nun an widmete sich Rzewuski nicht nur der weiteren Erforschung der Röntgenstrahlen. In eigentlichen Röntgenkursen machte er die Ärzte des Kantons und in öffentlichen Demonstrationen und Vorträgen ein weiteres Publikum mit den wunderbaren Möglichkeiten der Röntgenstrahlen bekannt.

Die Davoser Blätter schreiben am 5. September 1896 nach einem Hinweis auf den eben erwähnten ersten öffentlichen Vortrag:

«Herr Rzewuski hat auch seither die Fortschritte, die auf diesem neuen Gebiet gemacht worden sind, eifrig verfolgt und sich die zum Experimentieren notwendigen Instrumente angeschafft.

Am vorigen Samstag hatte er nun sämtliche in Davos weilenden Ärzte zu sich eingeladen, um ihnen die Ergebnisse und den Stand der bezüglichen Forschung ad oculos zu demonstrieren.» Diesmal begnügte er sich nicht mit der Demonstration von Knochenaufnahmen, sondern zeigte an einem 12 jährigen Knaben, «daß die Bewegungen des Zwerchfells beim Atmen sowie die Schläge des Herzens wohl erkennbar sind, wenn auch nicht in so ausgeprägter Weise, daß sie z.B. für die Diagnose eines Herzfehlers von Bedeutung sein könnten.»

Mit der Zeit wandelte Rzewuski sein Laboratorium in ein Röntgenkabinett um. Dessen Einrichtung kam während des Zweiten Weltkrieges durch Vermittlung von Professor K. von Neergando in das medizin-historische Museum der Universität Zürich (20b). Auch übernahm Rzewuski die Ein- 
richtung von Röntgenlaboratorien in verschiedenen Sanatorien in Davos, wo 1910 nach dem Jahresbericht des EwD bereits 27 Röntgenapparate in Betrieb waren. Durch seine Verfeinerung der Aufnahmetechnik hat er der Tuberkulosediagnostik in Davos große Dienste geleistet (23).

Nach Dr.Oskar Bernhard, Spitalarzt in Samedan und Begründer der Heliotherapie bei chirurgischer Tuberkulose, stellte der bescheidene Röntgenpionier aus Davos sein technisches Geschick und seine Einrichtungen auch dem übrigen Kanton zur Verfügung. Dieser Erfahrungsbericht - die erste klinische Originalarbeit über die medizinische Anwendung der Röntgenstrahlen im Correspondenzblatt für Schweizer Ärzte überhaupt (14) ist sehr aufschlußreich für die Anfangszeit und für den Einsatz der Beteiligten.

Im März 1897 reisten Rzewuski mit einer durch Bunsenbatterien gespeisten Röntgenröhre und Dr. Bernhard nach Poschiavo, um aus einem durch den Stich infizierten Kniegelenk eine Nadel zu entfernen, die schlußendlich ganz im Knochen des medialen Condylus lag. Auf der Rückkehr wurden in Samedan einige Patienten durchleuchtet, so ein Mädchen, das sich zweieinhalb Jahre zuvor eine feine Nadel in den linken Oberarm getrieben hatte. Die auf der Röntgenplatte sichtbaren vermeintlichen Nadelstücke wurden während der nachfolgenden Operation in Esmarchscher Blutleere aber nicht gefunden. "Diese Sache», so schreibt Dr. Bernhard weiter, «ließ mich aber nicht ruhen, und als Herr Rzewuski im Juli zu Röntgenvorträgen wieder ins Engadin kam, ließ ich das Mädchen abermals durchleuchten, und was sahen wir? Die beiden Nadelstücke vollkommen wie vor der Operation, da wo ... die Narbe saß, und wo meine Finger das ganze Gebiet wiederholt abgetastet hatten. » Der Chirurg nahm an, die Nadel sei oxydiert und der Kern bereits resorbiert gewesen und fragt sich nun: «Gibt Eisenrost dieselbe scharfe Nuancierung wie eine kompakte Stahlnadel selbst?». Und nun ruhen beide nicht, bis diese Frage qualitativ und quantitativ gelöst ist.

Bei den experimentellen Untersuchungen war ihnen auch Professor Nussberger, Physiklehrer an der Kantonsschule in Chur, behilflich. Hier war nämlich auf ein Gesuch des Churer Ärztevereins durch Kleinratsbeschluß vom 29. Januar 1897 ein Röntgenzimmer eingerichtet worden, dessen Benützung allen Ärzten offenstand (25).

Überblickt man die Anfänge der Radiologie, so ist man einerseits erstaunt über die Schnelligkeit, mit der die Entdeckung Röntgens zu Experimenten und Publikationen in der ganzen Welt und auch bei uns Anlaß gab (22). Dies mag zum Teil dadurch bedingt sein, daß Röntgen auf eine Patentierung seiner Entdeckung verzichtete, andererseits aber auch darum, weil die notwendigen, relativ einfachen Apparaturen (Induktionsspulen und Vakuumröhren) in den neunziger Jahren des letzten Jahrhunderts in 
vielen physikalischen Laboratorien bereits vorhanden waren. Im weiteren mag vor allen Dingen die praktische Anwendbarkeit in der medizinischen Diagnostik, die auch von Laien schon sehr früh erkannt worden war, die explosive Entwicklung der Radiologie in ihren Anfängen mitbedingt haben. Erstaunlich bleibt andererseits der Mangel an Skepsis gegenüber einer Methode, die offenbar gerade dadurch, daß sie den menschlichen Sinnen nicht direkt zugänglich ist, paradoxerweise als besonders glaubwürdig angesehen wurde, eine Einstellung, die heute noch weit verbreitet ist.

Auch die Faszination des «Durchschauens» des menschlichen Körpers und der Glaube an das morphologische Substrat einer jeden Erkrankung haben sich seit dieser Zeit erhalten.

Bald wurden die ersten Röntgenschäden bekannt, die zur Vorsicht mahnten. Wie andere Röntgenpioniere aus dieser ersten Zeit, hat auch Rzewuski seinen wissenschaftlichen Eifer mit schweren Verbrennungen an beiden Händen büßen müssen (23).

Abschließend sei noch die isolierte Meinung eines mißgünstigen Kollegen aus der Pionierzeit der Röntgenologie erwähnt, der in einem Lokalblatt des Kantons Neuenburg 1898 die Meinung vertrat, die Anwendung der X-Strahlen sei eine «fumisterie allemande», um die nur deshalb soviel «Tamtam» gemacht werde, weil der deutsche Kaiser sich auf allen Gebieten eine Geschichte schaffen wolle und deshalb wünsche, daß in späteren Zeiten erzählt werde, die Entdeckung sei unter seiner Regierung erfolgt (15).

Das große Interesse der Öffentlichkeit für Röntgens Entdeckung und die rasche Anwendung der Röntgenstrahlen in der medizinischen Diagnostik in Graubünden ist in erster Linie Rzewuskis Verdienst.

Auch an der weiteren Entwicklung der technischen Grundlagen der Radiologie (die nicht in den Rahmen dieses kleinen Aufsatzes fällt) hatte der bescheidene Privatgelehrte, der 1918 Ehrenbürger von Davos wurde, Anteil.

\section{Publikationen von Alexander Rzewuski}

Chemische Wirkung der X-Strahlen, Naturwiss. Rdsch. 11 (1896) 419-420.

Ein elektrolytischer Unterbrecher für schwache Ströme, Annalen der Physik 1 (1900) 614-616.

Zur Röntgenographie des Thorax dyspnoischer Patienten bei Atemstillstand, Breuer's Beiträge zur Klinik der Tuberkulose, Band X, Heft 1 (1908).

Wechselstrom und Grätzsche Zellen in der Röntgentechnik, Fortschritte auf dem Gebiete der Röntgenstrahlen, Band XVIII, Heft 1, S.61-64 (1911).

Eine neue Form der Härtemesser für Röntgenröhren nach dem Benoistschen Prinzip, 
Fortschritte auf dem Gebiete der Röntgenstrahlen, Band XIX, Heft 4, S.269-271 (1912).

Die Osmoregulierung, ein Mittel, um Röntgenröhren auf beliebige Härtegrade einzustellen, Fortschritte auf dem Gebiete der Röntgenstrahlen, Band XXVIII, S.253-255 (1918).

\section{Literaturverzeichnis}

Correspondenzblatt für Schweizer Ärzte:

Jg. XXVI (1896)

(1) S.127

(2) S.183-4.

(3) S.193 f.

(4) S.274.

(5) S.351.

(6) S.421-6.

(7) S.484-5.

(8) S.771.

(9) S. 775 .
Jg. XXVII (1897)

(10) S. 95.

(11) S.415.

(12) S.192.

(13) S.414.

(14) S.743-47.

Jg. XXVIII (1898)

(15) S.446.

(16) S. 725-7.

Jg. XXIX (1899)

(17) S.109-10.

(18) S. 596-97.

(19a) Davoser Blätter, 15.2.1896.

(19b) Davoser Blätter, 5.9.1896.

(20a) Ferdmann J., 60 Jahre in Davos, ein Jubiläum A. Rzewuskis, Davoser Revue 1939, Nr. 12.

(20b) Ferdmann J., Alexander Rzewuski als Pionier der Röntgenologie in der Schweiz, Davoser Revue 1943, Nr.3.

(21) Glaser Otтo, Wilhelm Conrad Röntgen, Springer-Verlag, Berlin/Göttingen/Heidelberg 1959.

(22) Lesky E., Die Wiener Medizinische Schule im 19.Jahrhundert, Graz/Köln 1965, S. 339-44.

(23) Neergatid v. K., Nienhaus E. und Zuppinger A., A. Rzewuski zum 80. Geburtstag. Schweiz. Med. Wschr. 71 (1941) 523.

(24) Nzz, Nr.10, vom 10.1.1896: Röntgens neuste optische Entdeckung.

(25) Wieser C., Über die Anfänge der Radiologie in Graubünden, in Äskulap in Graubünden, 1970, S. 355-362.

(26) ZaKovsky J., Die zwei ersten Veröffentlichungen zu Röntgens Entdeckung der neuen Strahlen (Mitteilung). 